\title{
Robustness of triple I algorithms based on Schweizer-Sklar operators in fuzzy reasoning
}

\author{
Minxia Luo* and Yaping Wang \\ Department of Information and Mathematics Sciences, China Jiliang University, China
}

Received: 11-August-2015; Revised: 04-November-2015; Accepted: 05-December-2015

(C)2016 ACCENTS

\begin{abstract}
In this paper, the perturbation of fuzzy connectives and the robustness of fuzzy reasoning are investigated. This perturbation of Schweizer-Sklar parameterized t-norms and its residuated implication operators are given. We show that full implication triple I algorithms based on Schweizer-sklar operators are robust for normalized Minkowski distance.
\end{abstract}

\section{Keywords}

Schweizer-Sklar operators, Triple I algorithms, Fuzzy reasoning, Robustness.

\section{Introduction}

Since Zadeh [18] proposed the concept of fuzzy sets in 1965, the fuzzy theory is widely used in mathematics and many other application fields. As for fuzzy reasoning, the most basic models are given as follows (see [16, 22]):

Given the input "x is $A^{*}$ " and fuzzy rule "if $\mathrm{x}$ is $\mathrm{A}$ then $y$ is B", try to deduce a reasonable output " $y$ is $B^{*}$, fuzzy modus ponens (FMP);

Given the input "y is $B^{*}$ " and fuzzy rule "if $\mathrm{x}$ is $\mathrm{A}$ then $\mathrm{y}$ is $\mathrm{B}$ ", try to deduce a reasonable output " $x$ is $A^{*}$, fuzzy modus tollens (FMT).

Zadeh [19, 20, 21] introduce an influential approach called compositional rule of inference (CRI method) to deal with FMP and FMT. But CRI method lacks solid logical basis and has some arbitrariness.

In 1999, Wang [12, 13] propose a triple I method for fuzzy reasoning based on $\mathrm{R}_{0}$-implication operator by combining fuzzy logic and fuzzy reasoning, establishing the triple I principles for the models FMP and FMT.

\footnotetext{
*Author for correspondence

This work is supported by the National Natural Science

Foundation of China (No. 61273018 and 61302190).

Wang and Fu [15] established full implication triple I inference algorithms based on regular implications and normal implications. Pei [10] discuss the full implication inference for all residuated implication introduced by left continuous t-norms to solve FMP and FMT problem. In addition, Pei [11] conducted a detailed research into the triple I algorithms based on the monoidal t-norm basic logical system MTL setting a sound logic foundation. Furthermore, Luo and Yao [8] studied the full implication triple I algorithms based on Schweizer-Sklar parameterized family of t-norms and the continuity of this algorithm.

In fuzzy control, practical fuzzy reason schemes are likely to be perturbed by various types of noise. Therefore, the robustness of fuzzy inference algorithms is important. Cai [1] analyzed the robustness based on measuring the errors of consequents produced by the errors of premises in fuzzy reasoning based on equalities. Dai et al. [2] discuss the perturbation of some t-norms and corresponding residuated implication operators, and the robustness of the CRI solution for the FMP and FMT model. Subsequently [3] investigate the degree of equality of fuzzy sets and some important fuzzy implications and the robustness of triple I methods for fuzzy reasoning. $\mathrm{Li}$ et al. [9] studied the robustness of fuzzy reasoning by a concept similar to the modulus of continuity.

In this paper, the perturbation of Schweizer-Sklar operators and the robustness of triple I algorithms 
based on Schweizer-Sklar operators are investigated based on approximately equal under Minkowski distance. First, we review the necessary definitions and some lemmas relate to this paper. In section 3, the perturbation of Schweizer-Sklar operators is studied. In section 4, the robustness of triple I algorithms based on Schweizer-Sklar operators is discussed.

\section{Preliminaries}

In this section, the definitions of Schweizer-Sklar tnorms, their residuated implication operators and the solution of parameterized triple I algorithms are reviewed; in addition, some necessary lemmas are listed.

Definition 1 ([5, 7, 14]) The Schweizer-Sklar parameterized family of t-norms is a function

$T_{m}:[0,1]^{2} \rightarrow[0,1]$, for all $x, y \in[0,1]$ and $m \in R$,

$$
T_{m}(x, y)=\left\{\begin{array}{l}
\left(\max \left(0, x^{m}+y^{m}-1\right)\right)^{\frac{1}{m}}, m \in(-\infty, 0) \cup(0, \infty) ; \\
x y, \quad m=0\left(\text { the product } T_{P}\right) ; \\
\min \{x, y\}, \quad m=-\infty\left(\text { the } \min \text { imum } T_{G}\right) ; \\
\left\{\begin{array}{l}
\min \{x, y\}, x \wedge y=1 \\
0, \quad \text { otherwise }
\end{array}, m=\infty\right. \\
\left(\text { drastic product } T_{D}\right) .
\end{array}\right.
$$

Definition 2 ([5, 14]) The Schweizer-Sklar family of residuated implication operators induced by parameterized $\mathrm{t}$-norms is also a function $I_{m}:[0,1]^{2} \rightarrow[0,1]$, for all $x, y \in[0,1]$ and $m \in R$,

$I_{m}(x, y)=\left\{\begin{array}{l}\min \left\{1,\left(1-x^{m}+y^{m}\right)^{\frac{1}{m}}\right\}, m \in(-\infty, 0) \cup(0, \infty) ; \\ \min \left\{1, \frac{y}{x}\right\}, m=0 ; \\ \left\{\begin{array}{l}1, x \leq y \\ y, x>y\end{array}, m=-\infty\right. \\ \left\{\begin{array}{l}y, x=1 \\ 1, x \neq 1\end{array}, m=\infty .\right.\end{array}\right.$

Definition 3 ([6]) The normalized Minkowski distance of fuzzy sets $\mathrm{A}$ and $\mathrm{B}$ is

$$
d_{p}(A, B)=\sqrt[p]{\frac{1}{n} \sum_{i=1}^{n}\left|A\left(x_{i}\right)-B\left(y_{i}\right)\right|^{p}},
$$

where $p$ is a parameter satisfying.
Definition 4 ([2]) Let $U=\left\{x_{1}, x_{2}, \cdots \cdots x_{n}\right\}$ be a universe, $A$ and $\mathrm{B}$ be two fuzzy sets defined on $U$, and $\varepsilon \in[0,1]$. If

$$
d_{p}(A, B)=\sqrt[p]{\frac{1}{n} \sum_{i=1}^{n}\left|A\left(x_{i}\right)-B\left(x_{i}\right)\right|^{p}} \leq \varepsilon,
$$

then $\mathrm{A}$ and $\mathrm{B}$ are said to be $\varepsilon(p)$-approximately equal, denoted $A \square_{p}(\varepsilon) B$. Also, B is called an $\varepsilon(p)$-perturbation of $\mathrm{A}$.

We denote the residuated implication operators based on the Schweizer-Sklar t-norm by $\rightarrow_{m}$.

Lemma 1([8]) The $\rightarrow_{m}$ type triple I solution $B^{*}$ for FMP is given by the following formula: $\forall y \in Y$, when $m<\infty$,

$$
B^{*}(y)=\left\{\begin{array}{l}
\sup _{x \in E_{y}}\left\{\left(\left(A(x) \rightarrow_{m} B(y)\right)^{m}+\left(A^{*}(x)\right)^{m}-1\right)^{\frac{1}{m}}\right\}, \\
m \in(-\infty, 0) \cup(0, \infty) ; \\
\sup _{x \in E_{y}}\left\{\left(\left(A(x) \rightarrow_{p} B(y)\right) \cdot A^{*}(x)\right\}, m=0 ;\right. \\
\sup _{x \in E_{y}}\left\{A^{*}(x) \wedge\left(A(x) \rightarrow_{G} B(y)\right)\right\}, m=-\infty ;
\end{array}\right.
$$

Where

$$
E_{y}=\left\{\begin{array}{l}
\left.\left\{x \in X \mid\left(A(x) \rightarrow_{m} B(y)\right)^{m}+\left(A^{*}(x)\right)^{m}-1\right)>0\right\}, \\
m \in(-\infty, 0) \cup(0, \infty) ; \\
\left\{x \in X \mid A^{*}(x)>0 \text { and } A(x) \rightarrow_{m} B(y)>0\right\}, \\
m=0,-\infty .
\end{array}\right.
$$

when $m=\infty$

$$
B^{*}(y)=\left\{\begin{array}{l}
\sup _{x \in E_{y}}\left\{A^{*}(x) \wedge\left(A(x) \rightarrow_{D} B(y)\right)\right\}, \\
E_{y}=\left\{x \in X \mid A^{*}(x) \vee\left(A(x) \rightarrow_{D} B(y)\right)=1\right\} ; \\
0, \\
E_{y}=\left\{x \in X \mid A^{*}(x) \vee\left(A(x) \rightarrow_{D} B(y)\right) \neq 1\right\} .
\end{array}\right.
$$

Lemma 2([8]) The $\rightarrow_{m}$ type triple I solution $A^{*} B^{*}$ for FMT is given by the following formula: $\forall x \in X$, when $m<\infty$, 
International Journal of Advanced Computer Research, Vol 6(22)

$A^{*}(x)=\left\{\begin{array}{l}\inf _{y \in E_{x}}\left\{\left(1-\left(A(x) \rightarrow_{m} B(y)\right)^{m}+\left(B^{*}(y)\right)^{m}\right)^{\frac{1}{m}}\right\}, \\ m \in(-\infty, 0) \cup(0, \infty) ; \\ \inf _{y \in E_{x}}\left\{\frac{B^{*}(y)}{A(x) \rightarrow_{p} B(y)}\right\}, m=0 ; \\ \inf _{y \in E_{x}}\left\{B^{*}(y)\right\}, \quad m=-\infty ;\end{array}\right.$
where $\quad E_{x}=\left\{y \in Y \mid B^{*}(y)<A(x) \rightarrow_{m} B(y), m<\infty\right\} ;$
$A^{*}(x)=\left\{\begin{array}{l}\inf _{y \in E_{x}}\left\{B^{*}(y)\right\}, E_{x}=\left\{y \in Y \mid A(x) \rightarrow_{m} B(y)=1\right\} ; \\ 1, E_{x}=\left\{y \in Y \mid A(x) \rightarrow_{m} B(y) \neq 1\right\} .\end{array}\right.$

Lemma 3 ([4]) Let $x, y>0$ and $x \neq y$, then

$r x^{r-1}(x-y)>x^{r}-y^{r}>r y^{r-1}(x-y)(r<0$ or $r>1)$

$r x^{r-1}(x-y)<x^{r}-y^{r}<r y^{r-1}(x-y) \quad(0<r<1)$

$r x^{r-1}(x-y)=x^{r}-y^{r}=r y^{r-1}(x-y)$

$(r=0, r=1, x=y)$

Lemma 4 ([17]) Let I be a non-empty finite index set, then

$\left|\bigvee_{i \in I} a_{i}-\bigvee_{i \in I} b_{i}\right| \leq \underset{i \in I}{\vee}\left|a_{i}-b_{i}\right|, \quad\left|\bigwedge_{i \in I} a_{i}-\bigwedge_{i \in I} b_{i}\right| \leq \underset{i \in I}{\bigvee}\left|a_{i}-b_{i}\right|$

Lemma 5 Let $x, y \in[0,1], r>1$, then

$\left|x^{r}-y^{r}\right| \leq r|x-y|$

The proof is easy to carry out from Lemma 3 .

Lemma 6 Let the function

$f(x)=\left(a^{m}+x^{m}-1\right)^{1 / m}, x \in[c, b] \subset[0,1](c<b)$,

$a \in[0,1], m \geq 1, m \neq 0$, then $|f(b)-f(c)| \leq|b-c|$.

Lemma 6 is obtained from a proof of progress of Theorem 5 in paper [8].

Lemma 7 When $m \leq 1$ and $m \neq 0$, let two functions $g(x)=\left(1-a^{m}+x^{m}\right)^{1 / m} \quad$ and $h(x)=\left(1-x^{m}+a^{m}\right)^{1 / m}$, $g(x), h(x) \in[0,1], \quad a \in[0,1], \quad x \in[b, c] \subset[0,1]$, then $|g(b)-g(c)| \leq|b-c|$ and $|h(b)-h(c)| \leq|b-c|$.

The proof of Lemma 7 is similar to Lemma 6.
Lemma 8 (Minkowski's inquality) Let $\left(a_{1}, a_{2} \cdots a_{n}\right)$, $\left(b_{1}, b_{2} \cdots b_{n}\right) \in R^{n}$, and $1 \leq p<\infty$. Then $\left(\sum_{k=1}^{n}\left|a_{k}+b_{k}\right|^{p}\right)^{1 / p} \leq\left(\sum_{k=1}^{n}\left|a_{k}\right|^{p}\right)^{1 / p}+\left(\sum_{k=1}^{n}\left|b_{k}\right|^{p}\right)^{1 / p}$.

\section{Perturbation of Schweizer-Sklar operators}

Proposition 1 Suppose $d_{p}\left(A, A^{\prime}\right) \leq \varepsilon_{1}, d_{p}\left(B, B^{\prime}\right) \leq \varepsilon_{2}$ . $A$ and $A^{\prime}$ are two fuzzy sets defined on a finite universe contain $\mathrm{n}$ elements, $B$ and $B^{\prime}$ are two fuzzy sets defined on anther finite universe contain $\mathrm{k}$ elements, then we have

$d_{p}\left(T_{m}(A, B), T_{m}\left(A^{\prime}, B^{\prime}\right)\right) \leq$

$\varepsilon_{1}+\varepsilon_{2}, \quad m \in(-\infty, 0) \cup(0, \infty) ;$

$\varepsilon_{1}+\varepsilon_{2}, \quad m=0$

$\left\{\sqrt[p]{\left(\varepsilon_{1}^{p}+\varepsilon_{2}^{p}\right)}, \quad m=-\infty ;\right.$

$\sqrt[p]{\left(\varepsilon_{1}^{p}+\varepsilon_{2}^{p}\right)}, \quad m=\infty$

0 or 3 or 4 element in $\left\{a_{1 i}, a_{2 i}, b_{1 j}, b_{2 j}\right\}$ is 1 .

Proof: Suppose

$A=\left(a_{11}, a_{12}, \cdots a_{1 n}\right), A^{\prime}=\left(a_{21}, a_{22}, \cdots a_{2 n}\right)$,

$B=\left(b_{11}, b_{12}, \cdots b_{1 k}\right), B^{\prime}=\left(b_{21}, b_{22}, \cdots b_{2 k}\right)$

$C=T_{m}(A, B)=\left\{c_{i j}\right\}, i=1,2, \cdots n, j=1,2, \cdots k$.

$C^{\prime}=T_{m}\left(A^{\prime}, B^{\prime}\right)=\left\{c_{i j}^{\prime}\right\}, i=1,2, \cdots n, j=1,2, \cdots k$.

For $m>1$,

$$
\begin{aligned}
& \left|c_{i j}-c_{i j}^{\prime}\right|=\left|\left(a_{1 i}^{m}+b_{1 j}^{m}-1\right)^{1 / m}-\left(a_{2 i}^{m}+b_{2 j}^{m}-1\right)^{1 / m}\right| \\
& \leq \frac{1}{m}\left|\left(a_{1 i}^{m}+b_{1 j}^{m}-1\right)-\left(a_{2 i}^{m}+b_{2 j}^{m}-1\right)\right|(\text { Lemma } 5) \\
& \leq \frac{1}{m}\left(\left|a_{1 i}^{m}-a_{2 i}^{m}\right|+\left|b_{1 j}^{m}-b_{2 j}^{m}\right|\right) \\
& \leq \frac{1}{m}\left(\left|m\left(a_{1 i}-a_{2 i}\right)\right|+\left|m\left(b_{1 j}-b_{2 j}\right)\right|\right)(\text { Lemma } 5) \\
& =\left|a_{1 i}-a_{2 i}\right|+\left|b_{1 j}-b_{2 j}\right| .
\end{aligned}
$$

For $m \leq 1$ and $m \neq 0$, 
Minxia Luo et al.

$$
\begin{aligned}
& \left|c_{i j}-c_{i j}^{\prime}\right|=\left|\left(a_{1 i}^{m}+b_{1 j}^{m}-1\right)^{1 / m}-\left(a_{2 i}^{m}+b_{2 j}^{m}-1\right)^{1 / m}\right| \\
& =\left|\begin{array}{l}
\left(a_{1 i}^{m}+b_{1 j}^{m}-1\right)^{1 / m}-\left(a_{1 i}^{m}+b_{2 j}^{m}-1\right)^{1 / m} \\
+\left(a_{1 i}^{m}+b_{2 j}^{m}-1\right)^{1 / m}-\left(a_{2 i}^{m}+b_{2 j}^{m}-1\right)^{1 / m}
\end{array}\right| \\
& \leq\left|\left(a_{1 i}^{m}+b_{1 j}^{m}-1\right)^{1 / m}-\left(a_{1 i}^{m}+b_{2 j}^{m}-1\right)^{1 / m}\right| \\
& +\left|\left(a_{1 i}^{m}+b_{2 j}^{m}-1\right)^{1 / m}-\left(a_{2 i}^{m}+b_{2 j}^{m}-1\right)^{1 / m}\right| \\
& \leq\left|b_{1 j}-b_{2 j}\right|+\left|a_{1 i}-a_{2 i}\right|(\text { Lemma } 6) \text {. }
\end{aligned}
$$

Thus we obtain

$$
\begin{aligned}
& d_{p}\left(T_{m}\left(A, A^{\prime}\right), T_{m}\left(B, B^{\prime}\right)\right) \\
& =\sqrt[p]{\frac{1}{n k} \sum_{i=1}^{n} \sum_{j=1}^{k}\left|c_{i j}-c_{i j}^{\prime}\right|^{p}} \\
& \leq \sqrt[p]{\frac{1}{n k} \sum_{i=1}^{n} \sum_{j=1}^{k}\left(\left|a_{1 i}-a_{2 i}\right|+\left|b_{1 j}-b_{2 j}\right|\right)^{p}} \\
& \leq \sqrt[p]{\frac{1}{n k} \sum_{i=1}^{n} \sum_{j=1}^{k}\left(\left|a_{1 i}-a_{2 i}\right|^{p}\right.}+ \\
& \sqrt[p]{\frac{1}{n k} \sum_{i=1}^{n} \sum_{j=1}^{k}\left(\left|b_{1 j}-b_{2 j}\right|^{p}\right.}(\text { Lemma 8) } \\
& \leq \varepsilon_{1}+\varepsilon_{2}
\end{aligned}
$$

For $\mathrm{m}=0$,

$$
\begin{aligned}
& d\left(T_{m}\left(A, A^{\prime}\right), T_{m}\left(B, B^{\prime}\right)\right) \\
& =\sqrt[p]{\frac{1}{n k} \sum_{i=1}^{n} \sum_{j=1}^{k}\left|c_{i j}-c_{i j}^{\prime}\right|^{p}} \\
& =\sqrt[p]{\frac{1}{n k} \sum_{i=1}^{n} \sum_{j=1}^{k}\left|a_{1 i} \cdot b_{1 j}-a_{2 i} \cdot b_{2 j}\right|^{p}} \\
& =\sqrt[p]{\frac{1}{n k} \sum_{i=1}^{n} \sum_{j=1}^{k}\left|a_{1 i} \cdot b_{1 j}-a_{1 i} \cdot b_{2 j}+a_{1 i} \cdot b_{2 j}-a_{2 i} \cdot b_{2 j}\right|^{p}} \\
& =\sqrt[p]{\frac{1}{n k} \sum_{i=1}^{n} \sum_{j=1}^{k}\left(a_{1 i}\left|b_{1 j}-b_{2 j}\right|+b_{2 j}\left|a_{1 i}-a_{2 i}\right|\right)^{p}} \\
& \leq \sqrt[p]{\frac{1}{n k} \sum_{i=1}^{n} \sum_{j=1}^{k}\left(\left|b_{1 j}-b_{2 j}\right|+\left|a_{1 i}-a_{2 i}\right|\right)^{p}} \\
& \leq \varepsilon_{1}+\varepsilon_{2} \\
& \text { For } m=-\infty
\end{aligned}
$$

$$
\begin{aligned}
& d_{p}\left(T_{m}(A, B), T_{m}\left(A^{\prime}, B^{\prime}\right)\right) \\
& =\sqrt[p]{\frac{1}{n k} \sum_{i=1}^{n} \sum_{j=1}^{k}\left|c_{i j}-c_{i j}^{\prime}\right|^{p}} \\
& =\sqrt[p]{\frac{1}{n k} \sum_{i=1}^{n} \sum_{j=1}^{k}\left|\min \left\{a_{1 i}, b_{1 j}\right\}-\min \left\{a_{2 i}, b_{2 j}\right\}\right|^{p}} \\
& \leq \sqrt[p]{\frac{1}{n k} \sum_{i=1}^{n} \sum_{j=1}^{k} \max \left\{\left|a_{1 i}-a_{2 i}\right|,\left|b_{1 j}-b_{2 j}\right|\right\}^{p}}
\end{aligned}
$$

(Lemma 4)

$$
\begin{aligned}
& \leq \sqrt[p]{\frac{1}{n k} \sum_{i=1}^{n} \sum_{j=1}^{k}\left(\left|a_{1 i}-a_{2 i}\right|^{p}+\left|b_{1 j}-b_{2 j}\right|^{p}\right)} \\
& \leq \sqrt[p]{\left(\varepsilon_{1}^{p}+\varepsilon_{2}^{p}\right)}
\end{aligned}
$$

When $m=\infty$, if there is none element in $\left\{a_{1 i}, a_{2 i}, b_{1 j}, b_{2 j}\right\}$ is 1 , the result of the perturbation is 0 ; if there is one element in $\left\{a_{1 i}, a_{2 i}, b_{1 j}, b_{2 j}\right\}$ is 1 , the result of the perturbation is uncertain, we need to deal with situation like $\left|c_{i j}-c_{i j}^{\prime}\right|=b_{1 j}$; if there two elements in $\left\{a_{1 i}, a_{2 i}, b_{1 j}, b_{2 j}\right\}$ is 1 , the result of the perturbation is uncertain we need to deal with situation like $\left|c_{i j}-c_{i j}^{\prime}\right|=\left|a_{1 i}-b_{2 j}\right|$; if there three elements in $\left\{a_{1 i}, a_{2 i}, b_{1 j}, b_{2 j}\right\}$ is 1 , the result of the perturbation is $\sqrt[p]{\left(\varepsilon_{1}^{p}+\varepsilon_{2}^{p}\right)}$; if there four elements in $\left\{a_{1 i}, a_{2 i}, b_{1 j}, b_{2 j}\right\}$ is 1 , the result of the perturbation is 0 .

Remark 1 When $m=1$, the perturbation of Lukasiewicz t-norm is $\varepsilon_{1}+\varepsilon_{2}$.

Proposition 2 Suppose $\quad d_{p}\left(A, A^{\prime}\right) \leq \varepsilon_{1}$, $d_{p}\left(B, B^{\prime}\right) \leq \varepsilon_{2}, A$ and $A^{\prime}$ are two fuzzy sets defined on a finite universe contain n elements, $B$ and $B^{\prime}$ are two fuzzy sets defined on anther finite universe contain k elements, then we have 
International Journal of Advanced Computer Research, Vol 6(22)

$$
\begin{aligned}
& d_{p}\left(I_{m}(A, B), I_{m}\left(A^{\prime}, B^{\prime}\right)\right) \\
& \leq\left\{\begin{array}{l}
\varepsilon_{1}+\varepsilon_{2}, \quad m \in(-\infty, 0) \cup(0, \infty) ; \\
\frac{1}{a}\left(\varepsilon_{1}+\varepsilon_{2}\right), \quad m=0, \\
a=\min \left\{a_{1 i} \cdot a_{2 i}\right\}, i=1,2, \cdots n \\
\varepsilon_{2}, \quad m=-\infty, \\
a_{1 i}>a_{2 i} \text { and } b_{1 j}>b_{2 j} \text { or } a_{1 i}<a_{2 i} \text { and } b_{1 j}<b_{2 j} ; \\
\varepsilon_{2}, \quad m=\infty, A=1 \text { and } A^{\prime}=1 \text { or } A \neq 1 \text { and } A^{\prime} \neq 1 .
\end{array}\right.
\end{aligned}
$$

Proof: Suppose

$$
\begin{aligned}
& A=\left(a_{11}, a_{12}, \cdots a_{1 n}\right), A^{\prime}=\left(a_{21}, a_{22}, \cdots a_{2 n}\right), \\
& B=\left(b_{11}, b_{12}, \cdots b_{1 k}\right), B^{\prime}=\left(b_{21}, b_{22}, \cdots b_{2 k}\right) \\
& C=T_{m}(A, B)=\left\{c_{i j}\right\}, i=1,2, \cdots n, j=1,2, \cdots k ., \\
& C^{\prime}=T_{m}\left(A^{\prime}, B^{\prime}\right)=\left\{c_{i j}^{\prime}\right\}, i=1,2, \cdots n, j=1,2, \cdots k .
\end{aligned}
$$

For $m>1$,

$$
\begin{aligned}
& \left|c_{i j}-c_{i j}^{\prime}\right|=\left|\left(1-a_{1 i}^{m}+b_{1 j}^{m}\right)^{1 / m}-\left(1-a_{2 i}^{m}+b_{2 j}^{m}\right)^{1 / m}\right| \\
& \leq \frac{1}{m}\left|\left(1-a_{1 i}^{m}+b_{1 j}^{m}\right)-\left(1-a_{2 i}^{m}+b_{2 j}^{m}\right)\right|(\text { Lemma } 5) \\
& \leq \frac{1}{m}\left(\left|a_{1 i}^{m}-a_{2 i}^{m}\right|+\left|b_{1 j}^{m}-b_{2 j}^{m}\right|\right) \\
& \leq \frac{1}{m}\left(\left|m\left(a_{1 i}-a_{2 i}\right)\right|+\left|m\left(b_{1 j}-b_{2 j}\right)\right|\right)(\text { Lemma } 5) \\
& =\left|a_{1 i}-a_{2 i}\right|+\left|b_{1 j}-b_{2 j}\right| .
\end{aligned}
$$

For $m \leq 1$ and $m \neq 0$,

$\left|c_{i j}-c_{i j}^{\prime}\right|=\left|\left(1-a_{1 i}^{m}+b_{1 j}^{m}\right)^{1 / m}-\left(1-a_{2 i}^{m}+b_{2 j}^{m}\right)^{1 / m}\right|$

$=\mid\left(1-a_{1 i}^{m}+b_{1 j}^{m}\right)^{1 / m}-\left(1-a_{1 i}^{m}+b_{2 j}^{m}\right)^{1 / m}$

$+\left(1-a_{1 i}^{m}+b_{2 j}^{m}\right)^{1 / m}-\left(1-a_{2 i}^{m}+b_{2 j}^{m}\right)^{1 / m}$

$=\left|\left(1-a_{1 i}^{m}+b_{1 j}^{m}\right)^{1 / m}-\left(1-a_{1 i}^{m}+b_{2 j}^{m}\right)^{1 / m}\right|$

$+\left|\left(1-a_{1 i}^{m}+b_{2 j}^{m}\right)^{1 / m}-\left(1-a_{2 i}^{m}+b_{2 j}^{m}\right)^{1 / m}\right|$

$\leq\left|b_{1 j}-b_{2 j}\right|+\left|a_{1 i}-a_{2 i}\right|$ (Lemma 7).

Thus we obtain

$$
\begin{aligned}
& d_{p}\left(I_{m}\left(A, A^{\prime}\right), I_{m}\left(B, B^{\prime}\right)\right) \\
= & \sqrt[p]{\frac{1}{n k} \sum_{i=1}^{n} \sum_{j=1}^{k}\left|c_{i j}-c_{i j}^{\prime}\right|^{p}} \\
\leq & \sqrt[p]{\frac{1}{n k} \sum_{i=1}^{n} \sum_{j=1}^{k}\left(\left|a_{1 i}-a_{2 i}\right|+\left|b_{1 j}-b_{2 j}\right|\right)^{p}} \\
\leq & \sqrt[p]{\frac{1}{n k} \sum_{i=1}^{n} \sum_{j=1}^{k}\left(\left|a_{1 i}-a_{2 i}\right|^{p}\right.} \\
+ & \sqrt[p]{\frac{1}{n k} \sum_{i=1}^{n} \sum_{j=1}^{k}\left(\left|b_{1 j}-b_{2 j}\right|^{p}\right.}(\text { Lemma } 8) \\
\leq & \varepsilon_{1}+\varepsilon_{2} .
\end{aligned}
$$

For $\mathrm{m}=0$

$$
\begin{aligned}
& d_{p}\left(T_{m}(A, B), T_{m}\left(A^{\prime}, B^{\prime}\right)\right)=\sqrt[p]{\frac{1}{n k} \sum_{i=1}^{n} \sum_{j=1}^{k}\left|c_{i j}-c_{i j}^{\prime}\right|^{p}} \\
& =\sqrt[p]{\frac{1}{n k} \sum_{i=1}^{n} \sum_{j=1}^{k}\left|\min \left\{1, \frac{b_{1 j}}{a_{1 i}}\right\}-\min \left\{1, \frac{b_{2 j}}{a_{2 i}}\right\}\right|^{p}} \\
& \leq \sqrt[p]{\frac{1}{n k} \sum_{i=1}^{n} \sum_{j=1}^{k}\left(\frac{a_{2 i}\left|b_{2 j}-b_{1 j}\right|+b_{2 j}\left|a_{1 i}-a_{2 i}\right|}{a_{1 i} \cdot a_{2 i}}\right)^{p}} \\
& \leq \frac{1}{a} \sqrt[p]{\frac{1}{n k} \sum_{i=1}^{n} \sum_{j=1}^{k}\left(\left|b_{2 j}-b_{1 j}\right|+\left|a_{1 i}-a_{2 i}\right|\right)^{p}} \\
& \leq \frac{1}{a} \sqrt[p]{\frac{1}{n k} \sum_{i=1}^{n} \sum_{j=1}^{k}\left(\left|a_{1 i}-a_{2 i}\right|^{p}\right.}+ \\
& \frac{1}{a} \sqrt[p]{\frac{1}{n k} \sum_{i=1}^{n} \sum_{j=1}^{k}\left(\left|b_{1 j}-b_{2 j}\right|^{p}\right.}(\text { Lemma } 8) \\
& \leq \frac{1}{a}\left(\varepsilon_{1}+\varepsilon_{2}\right) \\
& a=\min \left\{a_{1 i} \cdot a_{2 i}\right\}, i=1,2, \cdots n
\end{aligned}
$$

For $\quad m=-\infty, \quad$ if $a_{1 i} \leq b_{1 j}, a_{2 i} \leq b_{2 j}$, then $d_{p}\left(c_{i j}, c_{i j}^{\prime}\right)=0$; $\quad$ if $\quad a_{1 i}>b_{1 j}, a_{2 i}>b_{2 j}$, then $d_{p}\left(I_{m}\left(A, A^{\prime}\right), I_{m}\left(B, B^{\prime}\right)\right) \leq \varepsilon_{2} ; \quad$ if $a_{1 i} \leq b_{1 j}, a_{2 i}>b_{2 j}$ or $a_{1 i}>b_{1 j}, a_{2 i} \leq b_{2 j}$, the result of $d_{p}\left(I_{m}\left(A, A^{\prime}\right), I_{m}\left(B, B^{\prime}\right)\right)$ is uncertain.

For $\quad m=\infty \quad$ if $\quad a_{1 i} \neq 1, a_{2 i} \neq 1$, then $d_{p}\left(I_{m}\left(A, A^{\prime}\right), I_{m}\left(B, B^{\prime}\right)\right)=0$; if $a_{1 i}=1, a_{2 i}=1$, then $d_{p}\left(I_{m}\left(A, A^{\prime}\right), I_{m}\left(B, B^{\prime}\right)\right) \leq \varepsilon_{2} ; \quad$ if $\quad a_{1 i} \neq 1, a_{2 i}=1$ or $a_{1 i}=1, a_{2 i} \neq 1$, the result of $d_{p}\left(I_{m}\left(A, A^{\prime}\right), I_{m}\left(B, B^{\prime}\right)\right)$ is uncertain.

Remark 2 When $m=1$, the perturbation of Lukasiewicz implication is $\varepsilon_{1}+\varepsilon_{2}$. 
Minxia Luo et al.

\section{Robustness of full implication triple I algorithms}

In this section, we will study the robustness of triple I algorithms based on Schweizer-Sklar parameterized operators.

Theorem 1 Suppose $d_{p}\left(A, A^{\prime}\right) \leq \varepsilon_{1}, d_{p}\left(B, B^{\prime}\right) \leq \varepsilon_{2}$, $d_{p}\left(A^{*}, A^{\prime *}\right) \leq \varepsilon_{3}, B^{*}$ and $B^{\prime *}$ are the $\rightarrow_{m}$-type triple I solutions for FMP, then

$$
\begin{aligned}
& d_{p}\left(B^{*}, B^{\prime *}\right) \leq \\
& \left\{\begin{array}{l}
\varepsilon_{1}+\varepsilon_{2}+\varepsilon_{3}, \quad m \in(-\infty, 0) \cup(0, \infty), \\
\left(A \rightarrow_{m} B\right)^{m}+\left(A^{*}\right)^{m}-1>0,
\end{array}\right. \\
& \left(A^{\prime} \rightarrow_{m} B^{\prime}\right)^{m}+\left(A^{\prime *}\right)^{m}-1>0 ; \\
& \left\{\frac{1}{a}\left(\varepsilon_{1}+\varepsilon_{2}\right)+\varepsilon_{3}, m=0\left(a=\min \left\{a_{1 i} \cdot a_{2 i}\right\}, i=1,2, \cdots n\right)\right. \\
& A^{*}>0 \text { and } A>B, A^{\prime^{*}}>0 \text { and } A^{\prime}>B^{\prime} \\
& \sqrt[p]{\left(\varepsilon_{2}^{p}+\varepsilon_{3}^{p}\right)}, \quad m=-\infty, \\
& A^{*}>0 \text { and } A>B, A^{\prime *}>0 \text { and } A^{\prime}>B^{\prime} \\
& \left\{\begin{array}{l}
\sqrt[p]{\left(\varepsilon_{2}^{p}+\varepsilon_{3}^{p}\right),} \quad m=\infty, \\
A^{*} \vee\left(A \rightarrow_{m} B\right)=1 \text { and } A^{\prime *} \vee\left(A^{\prime} \rightarrow_{m} B^{\prime}\right)=1 \\
0, \quad m=\infty, \\
A^{*} \vee\left(A \rightarrow_{m} B\right) \neq 1 \text { and } A^{\prime *} \vee\left(A^{\prime} \rightarrow_{m} B^{\prime}\right) \neq 1
\end{array}\right.
\end{aligned}
$$

Proof: For $m \in(-\infty, 0) \cup(0, \infty)$,

let $C=A \rightarrow_{m} B, C^{\prime}=A^{\prime} \rightarrow_{m} B^{\prime}$,

$$
\begin{aligned}
& \left(C^{m}+\left(A^{*}\right)^{m}-1\right)^{1 / m}=\left\{d_{q i}\right\}, q=1, \cdots, n k, i=1, \cdots, n, \\
& \left(C^{\prime m}+\left(A^{\prime *}\right)^{m}-1\right)^{1 / m}=\left\{d_{q i}^{\prime}\right\}, q=1, \cdots, n k, i=1, \cdots, n .
\end{aligned}
$$

Then we have $d_{p}\left(C, C^{\prime}\right) \leq \varepsilon_{1}+\varepsilon_{2}$ by Proposition 2 .

Moreover, we have

$$
\begin{aligned}
d_{p}\left(B^{*}, B^{\prime *}\right) & \leq \sqrt[p]{\frac{1}{n^{2} k} \sum_{q=1}^{n k} \sum_{i=1}^{n}\left|d_{q i}-d_{q i}^{\prime}\right|^{p}} \\
& \leq \varepsilon_{1}+\varepsilon_{2}+\varepsilon_{3}
\end{aligned}
$$

by Lemma1 、Proposition 1 and Lemma 4 .

When $\mathrm{m}=0$,

let $C=A \rightarrow_{m} B, C^{\prime}=A^{\prime} \rightarrow_{m} B^{\prime}$,

$\left(C \square A^{*}\right)=\left\{d_{q i}\right\}, q=1,2, \cdots, n k, i=1,2, \cdots, n$,

$\left(C^{\prime} \square A^{\prime *}\right)=\left\{d_{q i}^{\prime}\right\}, q=1,2, \cdots, n k, i=1,2, \cdots, n$.

Then we have $d_{p}\left(C, C^{\prime}\right) \leq \frac{1}{a}\left(\varepsilon_{1}+\varepsilon_{2}\right)$, $a=\min \left\{a_{1 i} \cdot a_{2 i}\right\}, i=1,2, \cdots n$ by Proposition 2 , and

$$
\begin{aligned}
d_{p}\left(B^{*}, B^{\prime *}\right) & =\sqrt[p]{\frac{1}{n^{2} k} \sum_{q=1}^{n k} \sum_{i=1}^{n}\left|d_{q i}-d_{q i}^{\prime}\right|^{p}} \\
& \leq \frac{1}{a}\left(\varepsilon_{1}+\varepsilon_{2}\right)+\varepsilon_{3}
\end{aligned}
$$

by Lemma1 and Proposition 1.

When $m=-\infty$, let $C=A \rightarrow_{m} B, C^{\prime}=A^{\prime} \rightarrow_{m} B^{\prime}$, $\left(C \wedge A^{*}\right)=\left\{d_{q i}\right\}, q=1,2, \cdots, n k, i=1,2, \cdots, n$, $\left(C^{\prime} \wedge A^{\prime *}\right)=\left\{d_{q i}^{\prime}\right\}, q=1,2, \cdots, n k, i=1,2, \cdots, n$.

Then we have $d_{p}\left(C, C^{\prime}\right) \leq \varepsilon_{2}$ by Proposition 2 , and

$$
\begin{aligned}
d_{p}\left(B^{*}, B^{\prime *}\right) & =\sqrt[p]{\frac{1}{n^{2} k} \sum_{q=1}^{n k} \sum_{i=1}^{n}\left|d_{q i}-d_{q i}^{\prime}\right|^{p}} \\
& \leq \sqrt[p]{\varepsilon_{2}^{p}+\varepsilon_{3}^{p}}
\end{aligned}
$$

by Lemma1 and Proposition 1.

When $m=\infty$,

let $C=A \rightarrow_{m} B C^{\prime}=A^{\prime} \rightarrow_{m} B^{\prime}$,

$\left(C \wedge A^{*}\right)=\left\{d_{q i}\right\}, q=1,2, \cdots, n k, i=1,2, \cdots, n$,

$\left(C^{\prime} \wedge A^{\prime *}\right)=\left\{d_{q i}^{\prime}\right\}, q=1,2, \cdots, n k, i=1,2, \cdots, n$

Then we have $d_{p}\left(C, C^{\prime}\right) \leq \varepsilon_{2}$ by Proposition 2 , and

$$
\begin{aligned}
d_{p}\left(B^{*}, B^{\prime *}\right) & =\sqrt[p]{\frac{1}{n^{2} k} \sum_{q=1}^{n k} \sum_{i=1}^{n}\left|d_{q i}-d_{q i}^{\prime}\right|^{p}} \\
& \leq \sqrt[p]{\varepsilon_{2}^{p}+\varepsilon_{3}^{p}}
\end{aligned}
$$

by Lemma1 and Proposition 1.

Theorem 2 Suppose $d_{p}\left(A, A^{\prime}\right) \leq \varepsilon_{1}, d_{p}\left(B, B^{\prime}\right) \leq \varepsilon_{2}$, $d_{p}\left(B^{*}, B^{\prime *}\right) \leq \varepsilon_{3}$ and $A^{*}$ and $A^{\prime *}$ are the $\rightarrow_{m}$-type triple I solution for FMT, then

$$
\begin{aligned}
& d_{p}\left(A^{*}, A^{\prime *}\right) \\
& \leq\left\{\begin{array}{l}
\varepsilon_{1}+\varepsilon_{2}+\varepsilon_{3}, m \in(-\infty, 0) \cup(0, \infty) ; \\
\frac{1}{b}\left(\varepsilon_{1}+\varepsilon_{2}+\varepsilon_{3}\right), m=0,\left(b=\min \left\{b_{1 j}, b_{2 j}\right\}, j=1,2, \cdots k ;\right) \\
\varepsilon_{3}, m=-\infty .
\end{array}\right.
\end{aligned}
$$

For $B^{*}<A \rightarrow_{m} B$ and $B^{\prime *}<A^{\prime} \rightarrow_{m} B^{\prime}$, when $m<\infty$, 
$d_{p}\left(A^{*}, A^{\prime *}\right)=\left\{\begin{array}{l}\varepsilon_{3}, \\ A \rightarrow_{m} B=1 \text { and } A^{\prime} \rightarrow_{m} B^{\prime}=1 \\ 0, \\ A \rightarrow_{m} B \neq 1 \text { and } A^{\prime} \rightarrow_{m} B^{\prime} \neq 1\end{array}\right.$

Proof: For $m \in(-\infty, 0) \cup(0, \infty)$,

let $C=A \rightarrow_{m} B, C^{\prime}=A^{\prime} \rightarrow_{m} B^{\prime}$,

$\left(1-C^{m}+\left(B^{*}\right)^{m}\right)^{1 / m}=\left\{d_{q i}\right\}, q=1, \cdots, n k, i=1, \cdots, n$,

$\left(1-C^{\prime m}+\left(B^{\prime *}\right)^{m}\right)^{1 / m}=\left\{d_{q i}^{\prime}\right\}, q=1, \cdots, n k, i=1, \cdots, n$.

Then we have $d\left(C, C^{\prime}\right) \leq \varepsilon_{1}+\varepsilon_{2}$ by Proposition 2,

and

$$
\begin{aligned}
d\left(A^{*}, A^{\prime *}\right) & \leq \sqrt[p]{\frac{1}{n^{2} k} \sum_{q=1}^{n k} \sum_{i=1}^{n}\left|d_{q i}-d_{q i}^{\prime}\right|^{p}} \\
& \leq \varepsilon_{1}+\varepsilon_{2}+\varepsilon_{3}
\end{aligned}
$$

by Lemma 2 , Proposition 2 and Lemma 4 .

When $\mathrm{m}=0$,

$$
\begin{aligned}
& \text { let } C=A \rightarrow_{m} B, C^{\prime}=A^{\prime} \rightarrow_{m} B^{\prime}, \\
& \left(\frac{B^{*}}{C}\right)=\left(\frac{B^{*}}{A \rightarrow B}\right)=\frac{A \cdot B^{*}}{B}=\left\{d_{q i}\right\}, q=1, \cdots, n k, i=1, \cdots, n \\
& \left(\frac{B^{\prime *}}{C^{\prime}}\right)=\left(\frac{B^{\prime *}}{A^{\prime} \rightarrow B^{\prime}}\right)=\frac{A^{\prime} \cdot B^{\prime *}}{B^{\prime}}=\left\{d_{q i}^{\prime}\right\}, q=1, \cdots, n k, i=1, \cdots, n
\end{aligned}
$$

Then we have $d\left(A \cdot B^{*}, A^{\prime} \cdot B^{\prime *}\right) \leq \varepsilon_{1}+\varepsilon_{3} \quad$ by

Proposition 1, and

$$
\begin{aligned}
& d\left(A^{*}, A^{\prime^{*}}\right)=\sqrt[p]{\frac{1}{n^{2} k} \sum_{q=1}^{n k} \sum_{i=1}^{n}\left|d_{q i}-d_{q i}^{\prime}\right|^{p}} \\
& \leq \frac{1}{b}\left(\varepsilon_{1}+\varepsilon_{2}+\varepsilon_{3}\right), \\
& b=\min \left\{b_{1 j}, b_{2 j}\right\}, j=1,2, \cdots k ;
\end{aligned}
$$

by Lemma 2 and Proposition 2.

When $m=-\infty$ and $m=\infty$, we have

$d_{p}\left(A^{*}, A^{\prime *}\right) \leq d_{p}\left(B^{*}, B^{\prime *}\right)=\varepsilon_{3}$ by Lemma 2 and

Lemma 4.

\section{Conclusion}

In this paper, the perturbation of Schweizer-Sklar parameterized family of operators was investigated. The robustness of triple I algorithms based on Schweizer-Sklar operators were studied. We proved that triple I algorithms based on Schweizer-Sklar tnorms have robustness for $m \in(-\infty, \infty)$, and robustness with special conditions for $m=-\infty$ and $m=\infty$. These conclusions will provide a reliable theoretical basis for fuzzy reasoning.

\section{Acknowledgment}

None.

\section{Conflicts of interest}

The authors have no conflicts of interest to declare.

\section{References}

[1] Cai KY. Robustness of fuzzy reasoning and $\delta$ equalities of fuzzy sets. IEEE Transactions on Fuzzy Systems. 2001; 9(5):738-50.

[2] Dai S, Pei D, Wang SM. Perturbation of fuzzy sets and fuzzy reasoning based on normalized Minkowski distances. Fuzzy Sets and Systems. 2012;189(1):6373.

[3] Dai S, Pei D, Guo D. Robustness analysis of full implication inference method. International Journal of Approximate Reasoning. 2013; 54(5):653-66.

[4] Hardy GH, Littlewood JE, Pólya G. Inequalities. Cambridge University Press; 1952.

[5] Huacan H, Hua W, Yonghuai L, Yongjun W, Yongwen D. Principle of universal logics. Science Press, Beijing; 2001.

[6] Hung WL, Yang MS. Similarity measures of intuitionistic fuzzy sets based on $\mathrm{Lp}$ metric. International Journal of Approximate Reasoning. 2007;46(1):120-36.

[7] Klement EP, Mesiar R, Pap E. Triangular norms. Position paper II: general constructions and parameterized families. Fuzzy Sets and Systems. 2004;145(3):411-38.

[8] Luo M, Yao N. Triple I algorithms based on Schweizer-Sklar operators in fuzzy reasoning. International Journal of Approximate Reasoning. 2013;54(5):640-52.

[9] Li Y, Qin K, He X. Robustness of fuzzy connectives and fuzzy reasoning. Fuzzy Sets and Systems. 2013;225:93-105.

[10] Pei D. Unified full implication algorithms of fuzzy reasoning. Information Sciences. 2008;178(2):520-30.

[11] Pei D. Formalization of implication based fuzzy reasoning method. International Journal of Approximate Reasoning. 2012;53(5):837-46.

[12] Wang GJ. The full implication triple I method of fuzzy reasoning. Science in China (Series E).1999;29(1):4353. (in Chinese).

[13] Wang GJ. On the logic foundation of fuzzy reasoning. Information Sciences. 1999;117(1):47-88.

[14] Whalen T. Parameterized R-implications. Fuzzy Sets and Systems. 2003;134(2):231-81.

[15] Wang GJ, Fu L. Unified forms of triple I method. Computers \& Mathematics with Applications. 2005;49(5):923-32. 
Minxia Luo et al.

[16] Wang GJ. Non-classical mathematical logic and approximate reasoning. Science Press, Beijing; 2008. (in Chinese).

[17] Xu WH, Xie ZK, Yang JY, Ye YP. Continuity and approximation properties of two classes of algorithms for fuzzy inference. Journal of Software. 2004;15(10):1485-92. (in Chinese).

[18] Zadeh LA. Fuzzy sets. Information and Control. 1965;8(3):338-53.

[19] Zadeh LA. The concept of a linguistic variable and its application to approximate reasoning-I. Information Sciences. 1975;8(3):199-249.

[20] Zadeh LA. The concept of a linguistic variable and its application to approximate reasoning-II. Information Sciences. 1975;8(4):301-57.

[21] Zadeh LA. The concept of a linguistic variable and its application to approximate reasoning-III. Information Sciences. 1975;9(1):43-80.

[22] Zadeh LA. Toward a generalized theory of uncertainty (GTU)-an outline. Information Sciences. 2005;172(1):1-40.

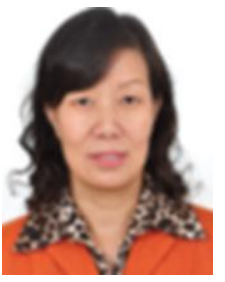

Minxia Luo received the Ph.D. degree in Computer Science from Northwestern Polytechnical University, China. She is now a Professor at Mathematics Department of the China Jiliang University, China. Her research fields are fuzzy logic theory, universal logic theory and artificial neural networks. She has published over 90 papers in international and domestic peer reviewed journals.

Email:minxialuo@163.com

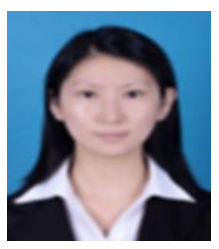

Yaping Wang received the M.Sc. degree in applied mathematics from China Jiliang University, China. Her research interests is fuzzy logic theory. 